that they did not need support at the time. 35 (88\%) of Paediatric trainees had not received any formal training on how to deal with many of these situations. All respondents agreed that further training on dealing with these issues was as important as their clinical training in paediatrics.

Conclusion Whilst many paediatric trainees felt well supported when dealing these events, many were not. It was evident that the School had to provide more robust, uniform support to all trainees. Since the survey dedicated monthly management seminars have been established, a mentoring scheme has been launched and specific legal training days have been offered. Consultants willing to offer such support have been signposted to trainees.

\section{G100 FEEDBACK IN PAEDIATRICS: IDENTIFYING ATTITUDES, PERCEPTIONS AND BARRIERS TO HIGH QUALITY EDUCATIONAL FEEDBACK WITHIN REGIONAL PAEDIATRIC TRAINING}

${ }^{1} \mathrm{~L}$ Gabbott, ${ }^{2} \mathrm{E}$ Osmond, ${ }^{3} \mathrm{~A}$ Baverstock. ${ }^{1}$ Paediatrics, Great Western Hospitals, Swindon, UK; ${ }^{2}$ Neonatal Intensive Care Unit, St Michael's Hospital, Bristol, UK; ${ }^{3}$ Paediatrics, Musgrove Park Hospital, Taunton, UK

\subsection{6/archdischild-2018-rcpch.97}

Paediatrics as a speciality has previously been a red outlier for the feedback category within the annual GMC training survey. This suggests that trainees at least, feel that feedback within their training is insufficient.

We wished to assess perceptions of feedback and identify both real and perceived barriers to successful feedback by sampling current trainees and supervisors within the Severn Deanery on their experiences.

The topic was introduced at a workshop during the Severn Deanery Paediatric Conference in 2016. Copies of the trainee questionnaire were distributed and subsequently an online version was sent via deanery email and the Severn Paediatrics Facebook group. Trainers were surveyed via an online form disseminated via email. We had a response rate of $65 \%$ for the trainee survey and $40 \%$ for the trainers' survey.

$72 \%$ of trainees reported feeling that they did not receive enough feedback, with several recurring themes identified, including lack of time, workload and staff shortages. This was also reflected in the trainers' survey but additionally the perception of feedback was cited as an issue, with trainees not always able to recognise 'feedback' in more informal situations, leading to a mismatch between the amount trainers felt was given versus the amount trainees acknowledged.

$83 \%$ of trainees wanted feedback integrated into daily practice, however only $34 \%$ of trainers felt that daily feedback was necessary. There was concern over trainee resilience and the ability to give constructive feedback to trainees; 'It does seem 'unfair' when they are busting a gut to keep the show on the road to give them any kind of negative feedback', however a recurring theme from trainees was to reduce the perceived stress of feedback by increasing its regularity; ' I think this should be normalised, i.e. recognising that there are always things we could do better, it's not a criticism'.

The barriers we identified to regular feedback are well recognised within current medical training. However, our responses suggest a cultural issue within paediatrics regarding the perceived resilience of paediatric trainees. Our next step is to challenge the culture of feedback locally to improve clinical training within our region.

\section{G101 JUNIOR DOCTOR LED TRAINING TO IMPROVE THE QUALITY OF QUALITY IMPROVEMENT}

${ }^{1} \mathrm{P}$ Rees, ${ }^{1} \mathrm{~J}$ Wright, ${ }^{2} \mathrm{~V}$ Jones. 'Gastroenterology, North Middlesex University Hospital, London, UK; ${ }^{2}$ Paediatrics, North Middlesex University Hospital, London, UK

\subsection{6/archdischild-2018-rcpch.98}

Aims All junior doctors are expected to undertake quality improvement (QI). However formal coaching in QI methods, their theoretical underpinning and application, is sporadic. This combination of mandatory QI with a lack of training can result in projects lacking theoretical underpinning and correct use of established methodology that fail to measurably or sustainably improve care, further demoralising trainees. We hypothesised that improving trainees' QI capability would increase their use of formal QI methods and subsequently improve the quality of QI at our institution. A six-week training programme was delivered to junior doctors by a junior doctor. We aimed to increase junior doctors' confidence using and applying the model for improvement.

Methods Training was aimed at junior doctors at an inner-city District General Hospital but open to multi-disciplinary staff. Teaching mirrored the Cincinnati Children's Qi algorithm, underpinned by Deming's theory of profound knowledge. This covered the QI process using the Model for Improvement. Sessions were didactic, interactive and practical, using real-life examples and repetition. Training was evaluated using the Kirkpatrick model with: knowledge assessments, evaluation forms and follow-up surveys. Sessions were adapted according to feedback. On completion, participants received certificates for their portfolios, the option of further coaching, and access to a QI toolkit.

Results Training was attended by 83 multidisciplinary staff: largely junior doctors, pharmacists and paediatric nurses. Knowledge, mapped to session aims, demonstrably increased. Average knowledge scores increased from $48 \%$ to $72.5 \%$ in week 1: and from $27 \%$ to $62 \%$ in week 6 . On completion, $81 \%$ felt confident using the Model for Improvement; 95\% felt confident completing a QI project. We received positive feedback about training being junior doctor-led and about the training programme. After training: 95\% felt they saw quality and safety issues differently, 95\% felt they had acquired new knowledge or skills and $100 \%$ said they felt empowered to improve.

Conclusion Junior doctors appreciated and benefitted from formal QI training. Since training, there are several on-going QI projects at our institution using the Model for Improvement. We hope build on this success by integrating QI training into junior doctors' curriculum at our hospital to further improve QI capability within this cohort of future leaders.

\section{G102 TOO MANY TWEETS, DON'T LIKE TO LIKE OR JUST LOST THE THREAD? THE HUB - A SINGLE PLATFORM FOR TRAINEE COMMUNICATION}

${ }^{1,2} \mathrm{~A}$ Lelliott, ${ }^{1,2} \mathrm{C}$ Holland, 1,2E Ambrose. 'ST4-8 Trainee Committee, Oxford School of Paediatrics, Oxford, UKi ${ }^{2}$ Children's Services, Oxford University Hospitals NHS Foundation Trust, Oxford, UK

\subsection{6/archdischild-2018-rcpch.99}

Aims Communication is key in healthcare, with poor communication linked to patient dissatisfaction and complaints. We believe that effective communication is equally important for 